\title{
Analyses of Pattern Formation Processes in Stochastic Activator-Inhibitor Systems with Saturation in Growth Domains
}

\author{
Masaaki Ishikawa and Keiichi Miyajima \\ Dept. of Computer Science and Systems Engineering, Yamaguchi University \\ 2-16-1 Tokiwadai, Ube, Yamaguchi 755-8611, Japan \\ E-mail: ishi@yamaguchi-u.ac.jp
}

\begin{abstract}
In the natural world, we often observe many kinds of spatio-temporal patterns such as patterns of animal coat, crystals of snow, etc. An analysis of generation processes of such patterns is very important as a basic problem in various fields of engineering including biological and material engineering. Among many spatiotemporal patterns, we especially study the pattern formation processes of sea shells from the viewpoint of biological engineering. As the mathematical model to generate the sea shells patterns, we consider an activatorinhibitor system, which is one of reaction diffusion systems. Taking into consideration the fact that some kind of disturbance exists to a greater or lesser degree in the natural world, we propose a stochastic activatorinhibitor system as the mathematical model of pattern formation processes of sea shells. We show that spatiotemporal patterns generated by the proposed model are robust for the random disturbance.
\end{abstract}

\section{Introduction}

In this paper, we study the pattern formation pro'cesses on the sea shells by the stochastic activatorinhibitor systems from the viewpoint of biological engineering [1]-[3]. The activator-inhibitor system considered here is one of reaction diffusion systems with two components, activator and inhibitor. The activatorinhibitor system can create complex patterns by the interaction between activator and inhibitor. One of characteristic aspects in analysis of pattern formations on sea shells is that sea shells grow with time, so that in modeling of generation of shell patterns, we must consider expansion of a spatial region due to growth in the model. Besides of expansion of the spatial region, there are several factors that determine the shell patterns such as width of stripes and figuration of patterns. As one of such factors, we can cite effect of saturation of the system state. From consideration above, we propose four types of models to create shell patterns, i.e., stochastic activator-inhibitor systems without saturation and ones with saturation in time-invariant or time-variant domains respectively.

By numerical simulations using the proposed models, the influence of saturation, expansion of the domain and the random noise on shell patterns is studied. And we show that shell patterns created by simulations are very similar to real shell pattern and shell patterns generated by the proposed model have robust property for random disturbance.

\section{Stochastic Activator-Inhibitor Systems without Saturation}

In this section, as the model to generate some kinds of shell patterns, we consider the activator-inhibitor systems without saturation of the system state in timeinvariant and time-variant domains. Letting $u(t, x)$ and $v(t, x)$ be concentrations of two substances, activator and inhibitor, at time $t$ and position $x$, consider the activator-inhibitor systems [1]:

$$
\begin{array}{r}
\frac{\partial u(t, x)}{\partial t}=d_{u} \Delta u(t, x)+s_{1}\left(\frac{u^{2}(t, x)}{v(t, x)}+b_{u}\right) \\
-r_{u} u(t, x), \quad(t, x) \in \Theta \times G \\
\frac{\partial v(t, x)}{\partial t}=d_{v} \Delta v(t, x)+s_{2} u^{2}(t, x)+b_{v} \\
-r_{v} v(t, x), \quad(t, x) \in \Theta \times G
\end{array}
$$

where $d_{u}$ and $d_{v}$ are diffusion coefficients of activator $u$ and inhibitor $v, \Delta(\cdot)$ denotes Laplacian, $s_{1}$ and $s_{2}$ are production rates, $r_{u}$ and $r_{v}$ are rates of removal and $b_{u}$ and $b_{v}$ are rates of basic production of activator $u$ and inhibitor $v$, respectively. And $\Theta=(0, T),(T$ is constant) and $G \subset R^{3}$ is a bounded open spatial region, we consider two kinds of $G$, one is time-invariant $G$ and the other is time-variant $G(t)$. Since shells grow with time, $G(t)$ is taken so as to be monotonically increasing in the time-variant case.

From Eqs.(1) and (2), we can see that the activator $u$ has a nonlinear autocatalytic influence and the activator promotes own production as well as one of the inhibitor $v$ and the production of activator is slowed down by the inhibitor $v$. Therefore, the concentration 
of both substances would reach a steady state, however, this steady state is locally unstable because a local increase of the activator will increase the activator further due to its autocatalytic property.

Initial conditions are given by

$$
u(0, x)=u_{0}(x), \quad v(0, x)=v_{0}(x), \quad x \in G .
$$

Assuming that there is no flow of the substances $u$ and $v$ through the boundary $\Gamma$ of $G$, we have boundary conditions as:

$$
\frac{\partial u(t, x)}{\partial \nu}=\frac{\partial v(t, x)}{\partial \nu}=0 \quad(t, x) \in \Theta \times \Gamma
$$

where $\partial(\cdot) / \partial \nu$ denote exterior normal derivative on the boundary $\Gamma$.

Taking into consideration the fact that some kinds of disturbance exists to a greater or lesser degree in the natural world, instead of the deterministic model (1) and (2), we propose a stochastic activator-inhibitor system as the mathematical model of pattern formation processes of sea shells:

$$
\begin{gathered}
\frac{\partial u(t, x)}{\partial t}=d_{u} \Delta u(t, x)+s_{1}\left(\frac{u^{2}(t, x)}{v(t, x)}+b_{u}\right) \\
-r_{u} u(t, x)+\alpha u \frac{\partial w(t, x)}{\partial t} \\
(t, x) \in \Theta \times G \\
\frac{\partial v(t, x)}{\partial t}=d_{v} \Delta v(t, x)+s_{2} u^{2}(t, x)+b_{v} \\
-r_{v} v(t, x)+\beta v \frac{\partial r(t, x)}{\partial t} \\
(t, x) \in \Theta \times G
\end{gathered}
$$

where $w(t, x)$ and $r(t, x)$ are mutually independent Wiener processes with respect to time, and their formal derivatives $\partial w(t, x) / \partial t$ and $\partial r(t, x) / \partial t$ denote the Gaussian white noises with respect to time respectively. The mathematical meaning of Eqs.(5) and (6) is given by stochastic integral equations:

$$
\begin{aligned}
u(t, x) & -\int_{0}^{t} d_{u} \Delta u(s, x) d s=u_{0}(x) \\
& +\int_{0}^{t} s_{1}\left(\frac{u^{2}(s, x)}{v(s, x)}+b_{u}\right) d s-\int_{0}^{t} r_{u} u(s, x) d s \\
& +\alpha \int_{0}^{t} u(s, x) d w(s, x) \quad(t, x) \in \Theta \times G \\
v(t, x)- & \int_{0}^{t} d_{v} \Delta v(s, x) d s=v_{0}(x) \\
& +\int_{0}^{t}\left(s_{2} u^{2}(s, x)+b_{v}\right) d s-\int_{0}^{t} r_{v} v(s, x) d s \\
& +\beta \int_{0}^{t} v(s, x) d r(s, x), \quad(t, x) \in \Theta \times G
\end{aligned}
$$

The boundary condition is given by Eq.(4).

\section{Stochastic Activator-Inhibitor Systems with Saturation}

There are several fractors to determine the shell patterns, i.e., width of stripes, distance between them and figuration of patterns. As one of such factors, the effect of saturation of the system state is cited. So, in this section, we consider the stochastic activator-inhibitor system with saturation. At high concentration of activator $u$, the reaction would slow down because of lack of available enzyme molecules. So, we consider the following stochastic activator-inhibitor system with saturation in concentration of activator $u$ :

$$
\begin{gathered}
\frac{\partial u(t, x)}{\partial t}=d_{u} \Delta u(t, x) \\
+s_{1}\left(\frac{u^{2}(t, x)}{v(t, x)\left(1+s_{u} u^{2}(t, x)\right)}+b_{u}\right) \\
-r_{u} u(t, x)+\alpha u(t, x) \frac{\partial w(t, x)}{\partial t} \\
(t, x) \in \Theta \times G \\
\frac{\partial v(t, x)}{\partial t}=d_{v} \Delta v(t, x)+s_{2} u^{2}(t, x)+b_{v} \\
-r_{v} v(t, x)+\beta v(t, x) \frac{\partial r(t, x)}{\partial t} \\
(t, x) \in \Theta \times G
\end{gathered}
$$

where $s_{u}$ is the saturation coefficient and meaning of the other parameters are same as Eqs.(1) and (2). It should be noted that the spatial region $G$ is taken to be time-invariant and monotonically increasing timevariant domain as Section 1.

The 2nd term of R.H.S. of (9) denotes the effect of saturation. Since the term $s_{u} u^{2}$ is negligible compared to 1 at low $u$-concentrations, Eqs.(9) and (10) have the same property as Eqs.(1) and (2) at low value of $u$. But with an increase in $u$, the term $s_{u} u^{2}$ becomes more dominant, so that self-enhancement by autocatalytic effect and suppression by saturation become the same order of $u^{2}$. Eventually, concentration of the activator has the upper bound.

The initial and boundary conditions are given by Eqs.(3) and (4).

The mathematical meaning of (9) and (10) is given by stochastic integral equations:

$$
\begin{aligned}
u(t, x) & -\int_{0}^{t} d_{u} \Delta u(s, x) d s=u_{0}(x) \\
& +\int_{0}^{t} s_{1}\left(\frac{u^{2}(s, x)}{v(s, x)\left(1+s_{u} u^{2}(s, x)\right)}+b_{u}\right) d s \\
& -\int_{0}^{t} r_{u} u(s, x) d s \\
& +\alpha \int_{0}^{t} u(s, x) d w(s, x),(t, x) \in \Theta \times G(11)
\end{aligned}
$$




$$
\begin{aligned}
v(t, x) & -\int_{0}^{t} d_{v} \Delta v(s, x) d s=v_{0}(x) \\
& +\int_{0}^{t}\left(s_{2} u^{2}(s, x)+b_{v}\right) d s \\
& -\int_{0}^{t} r_{v} v(s, x) d s+\beta \int_{0}^{t} v(s, x) d r(s, x) \\
(t, x) \in \Theta \times G & (1
\end{aligned}
$$

\section{Numerical Simulations}

In numerical simulations, we analyze the influence of the random noise, saturation in the system state and growth of the domain on the shell patterns.

\subsection{Simulations by Stochastic Activator In- hibitor Systems without Saturation \\ 4.1.1 Simulations in Time-invariant Domain}

In this section, we consider the stochastic activatorinhibitor system (5) and (6) without saturation in the time-invariant domain. Letting the spatial region $G$ be the one-dimensional time-invariant region $G=(0,100)$ and setting parameters in Eqs.(5) and (6) as below, results of simulations under the noise and the no noise are shown in Figs. 1 and 2 respectively:

Initial values: $u_{0}(x)=1.0+0.1 \xi(x), v_{0}(x)=0.5$

Diffusion coefficients: $d_{u}=0.01, d_{v}=0.4$

Removal rates: $r_{u}=0.02, r_{v}=0.06$

Basic production rates: $b_{u}=0.03, b_{v}=0$

Production rates: $s_{1}=s_{2}=0.02$

Coefficients of noises: $\alpha=\beta=0.03$

where $\xi(x)$ is normal random numbers on $G$.

Figures 1 and 2 show the time evolution of the concentration of the activator $u$ with the initial value $u_{0}$ with slight fluctuation around 1.0 under the noise and the no noise. And the color of region changes from white to black in proportion to concentration of $u$, for example, the white region shows the one with high concentration. In Figs. 1 and 2, time passes from top to bottom.

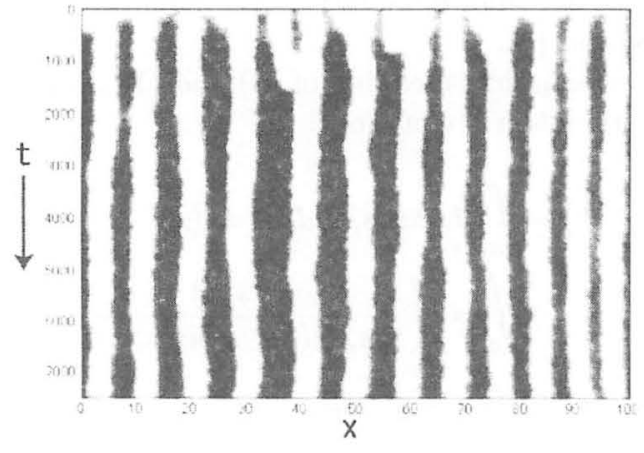

Fig. 1: Time evolution of $u(t, x)$ under the noise in the time-inavariant domain $G=(0,100)$

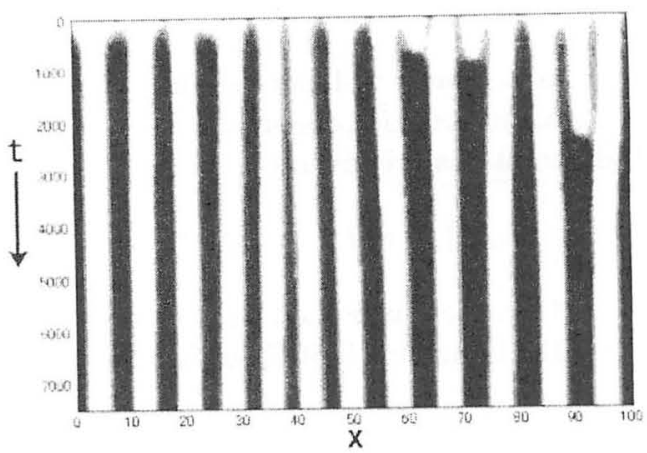

Fig. 2: Time evolution of $u(t, x)$ under the no noise in the time-invariant domain $G=(0,100)$

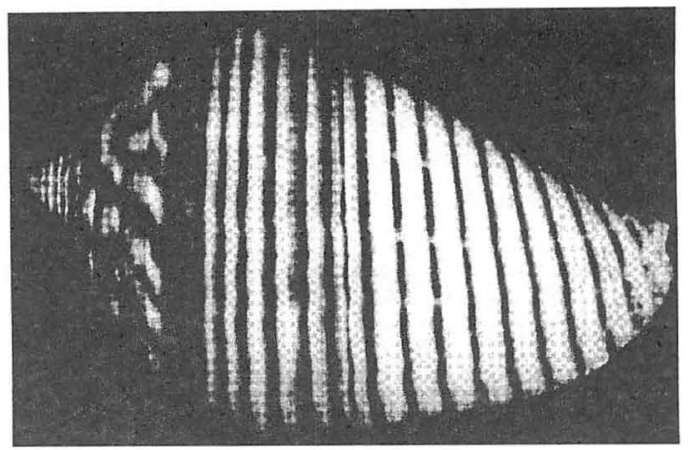

Fig. 3: Pattern on the shell (quoted from [1].)

As shown in Figs. 1 and 2, the stripes have different widths at irregular distances in both of the noise and the no noise cases. And, in the case with the noise, although stripes are slightly perturbed by the noise, the characteristics of the stripes as shown in Fig.2 are kept. Figure 3 shows a real pattern on the shell called Lyria Planicostana Taiwanica and we can see fluctuation of stripes on the shell. In the natural world, although there exist some kinds of fluctuation to a greater or lesser degree, the same species of shells have the similar patterns on the shell. This fact seems to mean that the shell patterns have robust property for the disturbance. Figures 1 and 2 show that this conjecture is plausible. And the pattern generated by the proposed model is very similar to the real one as Fig. 3, so that the proposed stochastic model is very useful for analysis of pattern formation processes on the shell.

\subsubsection{Simulations in Time-variant Domain}

In this section, we study the influence of growth of the shell on the shell pattern. Let the spatial region $G$ be the monotonically increasing time-variant region as $G(t)=(-0.0006 t, 20+0.0006 t)$ and setting the other parameters are same as Section 4.1.1, simulations are performed. Results of simulations under the noise and 
the no noise are shown in Figs. 4 and 5. And Figs.6 and 7 are $3 \mathrm{D}$ view of Figs. 4 and 5. From Figs. 4 to 7 , we can see that the generated pattern is different from one in the case of time-invariant region. With expansion of the spatial region, insertion of new stripes occurs in both of the noise and the no noise cases. Such insertion arises from the fact below. The distance between the activated regions increases because of growth of the domain, and the increase of the distance between the activated regions causes decreasing of the inhibitor concentration, so that the production of the activator begins. The generation of the activator means the insertion of new stripes. In the case with growth of the domain, the pattern has the robust property as well as the case of the invariant domain as shown in Figs. 4 to 8.

The insertion of new stripes is observed in a real shell pattern as shown in Fig. 8.

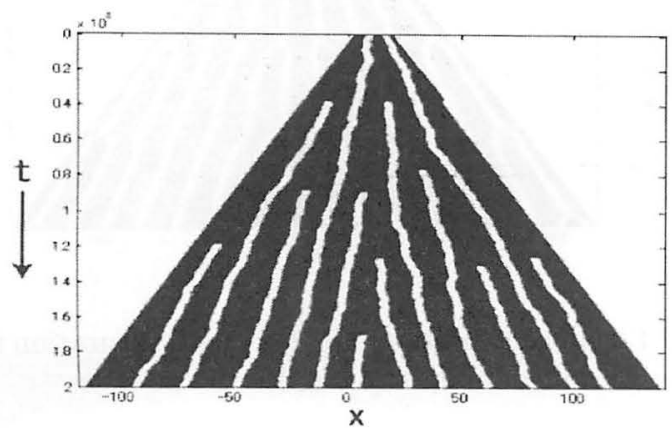

Fig. 4: Time evolution of $u(t, x)$ under the noise in the growth domain $G(t)$

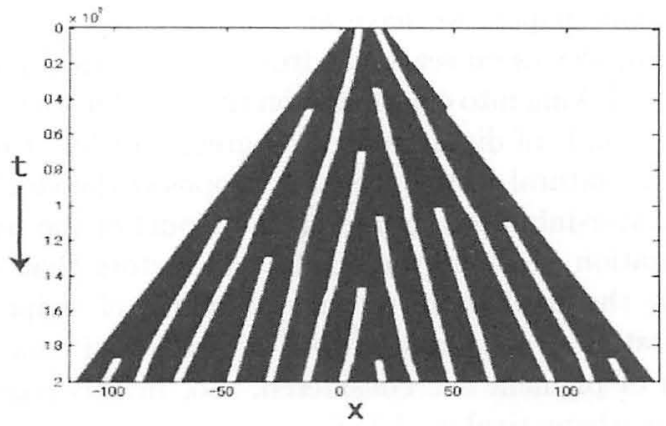

Fig. 5: Time evolution of $u(t, x)$ under the no noise in the growth domain $G(t)$

\subsection{Simulations by Stochastic Activator In- hibitor Systems with Saturation}

\subsubsection{Simulations in Time-invariant Domain}

In this section, we study the influence of saturation in the system state on the shell pattern using the proposed model (11) and (12) with time-inavriant and time-variant domains.

Setting the saturation parameter $s_{u}$ as $s_{u}=0.01$ and

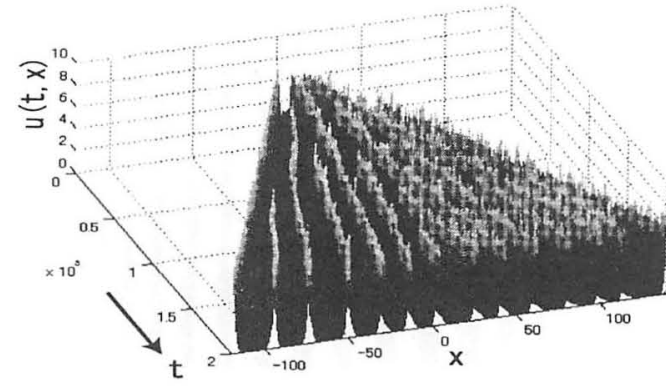

Fig. 6: 3D view of Fig. 4

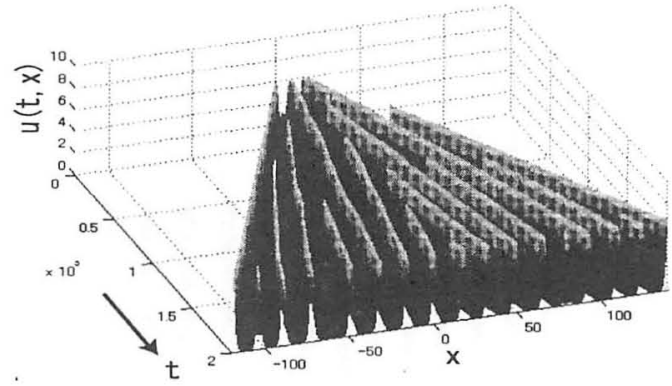

Fig. 7: 3D view of Fig. 5

other parameters are same as in Section 4.1.1, results of simulations are shown in Figs. 9 and 10. Figures 9 and 10 are time evolution of concentration of the activator $u$ under the noise and the no noise respectively. Comparing Figs. 1 and 2 obtained in the case without saturation with Figs. 9 and 10, we can see that there is little differene between them. So, the influence of saturation on the shell pattern seems to be little in the time-inavariant domain. And results of Figs. 9 and 10 show that the shell patterns have the robust property for the random noise.

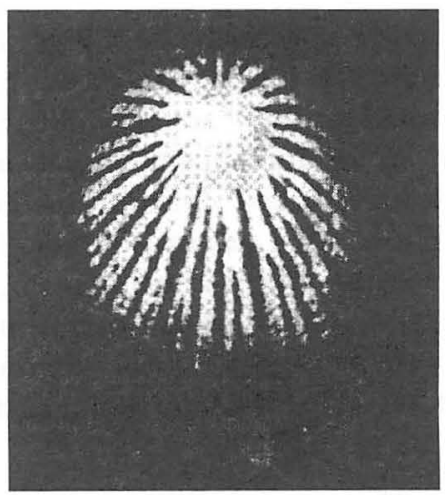

Fig. 8: Insertion of new lines on the shell (quoted from [1].) 


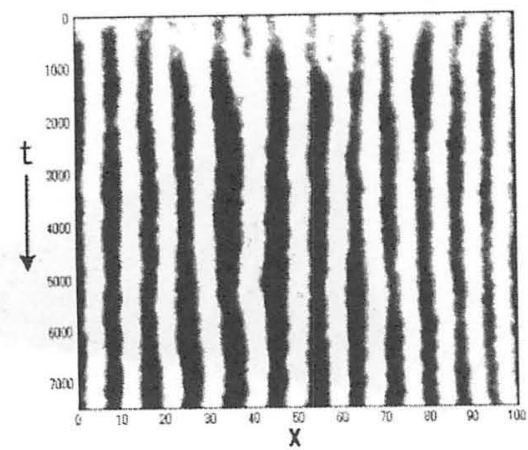

Fig. 9: Time evolution of $u(t, x)$ with saturation under the noise in the time-invariant domain $G=(0,100)$

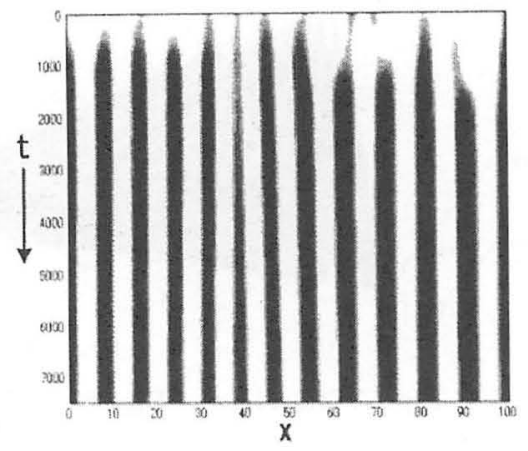

Fig. 10: Time evolution of $u(t, x)$ with saturation under the no noise in the time-invariant domain $G=(0,100)$

\subsubsection{Simulations in Time-variant Domain}

In this section, we consider the influence of saturation in the time-variant domain on the shell pattern. Setting the time-variant domain $G(t)$ as Section 4.1.2 and the other parameters are same as Section 4.2.1, simulations are performed. Results of simulations are shown in Figs. 11 and 12. Figures 11 and 12 are time evolution of concentration of the activator $u$ under the noise and the no noise respectively. And Figs. 13 and 14 are $3 \mathrm{D}$ view of Figs. 11 and 12. As shown in Figs. 11 to 14 , the generated patterns are very different from ones (Figs. 4 and 5) obtained in simulations without saturation in the time-variant domain. In the case where the activator $u$ has the saturation value in the monotonically increasing time-variant domain, insertion of stripes as Figs. 4 and 5 does not occur but bifurcation of stripes occurs as Figs. 11 to 14 . Figure 15 is a real shell pattern, on which the bifurcation of stripes can be observed.

Due to the existence of saturation value in activator $u$, width of stripes in the saturation region broadens, so that a split of stripe causes.

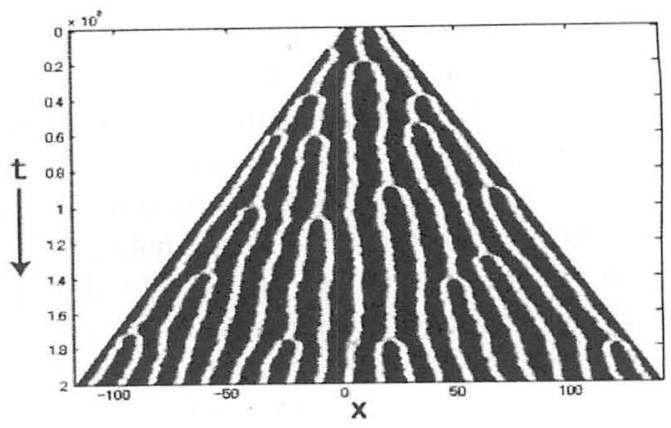

Fig. 11: Time evolution of $u(t, x)$ with saturation in the time-variant domain $G(t)$ under the noise

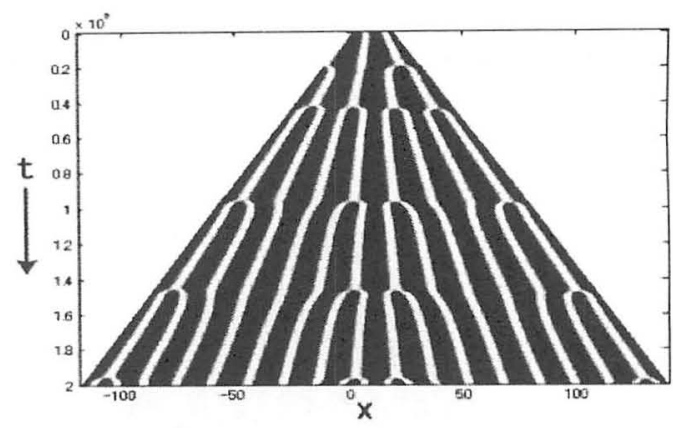

Fig. 12: Time evolution of $u(t, x)$ with saturation in the time-variant domain $G(t)$ under the no noise

\section{Conclusions}

In this paper, we have analyzed the pattern formation processes on sea shells from the biological point of view. Taking into consideration the fact that there exist some kinds of disturbance to a greater or lesser degree in the natural world, we have proposed the stochastic activator-inhibitor system as the model of the pattern formation processes on shells. As factors that determine the shell patterns such as width of stripes and figuration of patterns, growth of shells and concentration of pigment are considered. So, in this paper, as the mathematical model of the pattern formation on the shell, we have proposed four types of activator-inhibitor systems, i.e., systems with saturation and no saturation in concentration in the time-invariant and time-variant domains.

From numerical simulations, we have obtained results below:

(i) The shell patterns generated by the proposed models are robust for the random disturbance. This fact coincides with real phenomena because the same species of the shell have a similar pattern each other despite of the existence of some kinds of disturbance.

(ii) Effect of saturation of the concentration on the shell pattern is little in the case of time-invariant domain. 


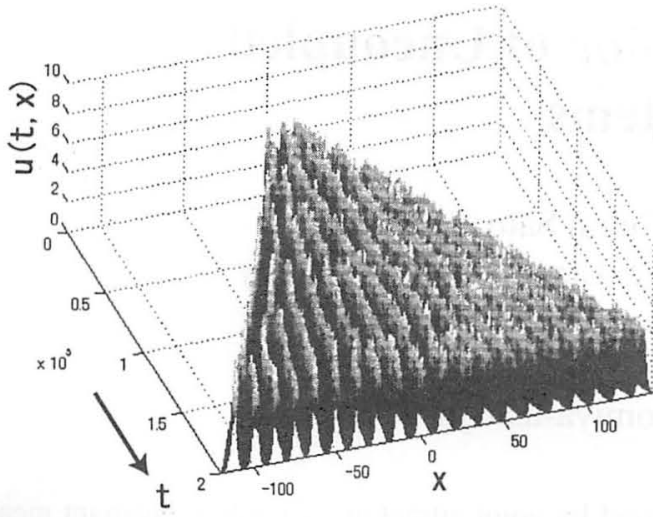

Fig. 13: 3D view of Fig. 11

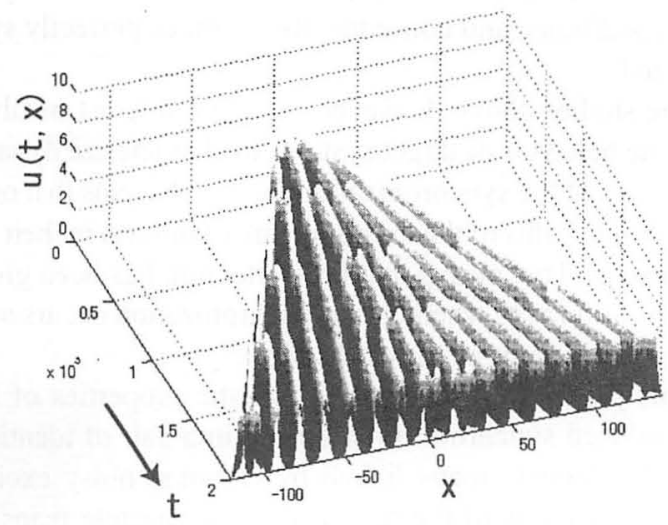

Fig. 14: 3D view of Fig. 12

(iii) Effect of growth of the shell on shell patterns is different by whether the concentration has saturation or not. Under the existence of saturation, stripes bifurcates with time, on the other hand, in the case of no saturation, insertion of new stripes occurs in the time-variant domain. As shown in Figs. 8 and 15, real shell patterns have insertion and bifurcation of stripes. In shell patterns generated by the proposed stochastic model are very similar to real ones, so the proposed model seems to be very useful in the analysis of pattern formation processes on shells.

Problems considered here are very important as basic problems of various fields of engineering, mainly because analyses of pattern formation processes in phase

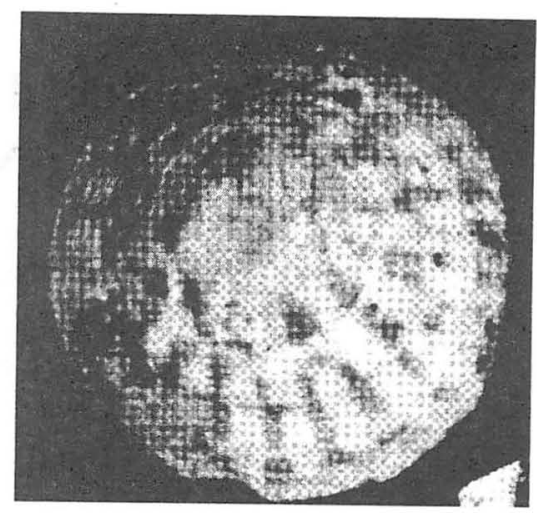

Fig. 15: Bifurcation of pattern on the shell (quoted from [1].)

transition of polymeric materials are essential to develop new materials and analyses of shell patterns are important from the viewpoint of making natural phenomena clear.

As the further problem to study, a qualitative analysis of influence of the random noise on the pattern formation processes is cited. A part of this work is supported by The Ministry of Education, Culture, Sports, Science and Technology under Grant-in-Aid for Research (C) (2)14550217.

\section{References}

[1] Hans Meinhardt: The Algorithmic Beauty of Sea Shells, Springer, Tubingen, 2000.

[2] Hans Meinhardt: Models of Biological Pattern Formation, Academic Press, 1982.

[3] J. D. Murray: Mathematical Biology I and II, Springer, 2003.

[4] J. D. Murray: "How the leopard gets the spots?", Scientific American., 258, pp. 80-87, 1988.

[5] D. S. Jones and B. D. Sleeman: Differential equations and Mathematical Biology, Chapman \& Hall/CRC, 2003.

[6] S. I. Rubinow: Introduction to Mathematical Biology, Dover Publication, 2002.

[7] D. Walgraef: Spatio-Temporal Pattern Formation, Springer, 1997. 